\title{
Optimistic and realistic perspectives on cognitive biases.
}

\author{
Pete C. Trimmer
}

University of California, Department of Environmental Science and Policy, 1 Shields Avenue, Davis, CA 95616, USA

Keywords: evolution, cognitive bias, signal detection, optimal behaviour, mental costs

\begin{abstract}
Numerous papers have tried to explain cognitive biases, such as optimism and overconfidence, from an evolutionary perspective. The attempts have met with mixed success. I identify why some approaches are more successful than others in explaining sub-optimal behaviours. I conclude that some evolutionary explanations of cognitive biases can be successful; the relevant explanation will depend on the particular bias being studied. In particular, I highlight the need to incorporate internal costs when considering the evolution of mental mechanisms, and how this can provide adaptive explanations of sub-optimal behaviours.
\end{abstract}

\section{Introduction and definitions}

Since Kahneman and Tversky pioneered the study of biases in cognitive processes (e.g., ${ }^{1}$ ), the topic has been of great interest to psychologists and, more recently, behavioural ecologists.

Table 1 supplies various definitions of the term 'cognitive bias'. The common theme is of a bias (or distortion) to a cognitive process or mental representation. A cognitive bias could result in optimal behaviour (i.e., behaviour that maximises expected payoff, often measured simply in terms of surviving offspring) according to some definitions but not others. These differences have led to confusion, discussed below. An additional layer of complexity emerges in lab experiments when an individual behaves according to adaptively evolved responses, but could increase rewards if the lab setting was understood by the subject.

In recent studies of non-human species, 'cognitive bias' has been used very generally, to mean decisions that are influenced by emotions $\left({ }^{2},{ }^{3},{ }^{4}\right)$, even if the consequent behaviours are optimal. This can be confusing, as a bias is arguably from something, and it is not always clear under this definition what a bias is from (functionally).

Raghubir \& Ranjan ${ }^{5}$ identify several stages at which apparent cognitive biases could arise, including: perception, memory-retrieval, information integration, making a judgement, and behaviour. Neuroscience is generally not yet able to directly identify distorted cognitive representations at a mechanistic level (though progress is being made; $6^{*}, 7,8$ ), so identification of a cognitive bias requires inference from behaviour (e.g., ${ }^{9 *}$ ). Note that the behaviour could simply be self-reporting of beliefs (although it is easy to mistakenly infer biases such as overconfidence from such reports ${ }^{10}$ ).

Many behaviours that initially appear irrational can be understood by taking background expectations and subsequent data into account from the perspective of the individual, as it is the expected payoff that determines whether a behaviour is optimal (rather than individual stochastic 
outcomes). The behavioural sciences have made great strides by using this 'optimality approach' to understand the distribution and timing of behaviours; e.g., the marginal value theorem ${ }^{11}$, driftdiffusion modelling ${ }^{12}$. However, not all behaviours are optimal; cognitive limitations mean that individuals will sometimes display sub-optimal cognitive biases even in their natural habitats. These behaviours are more challenging to explain from a theoretical perspective.

By dealing with sub-optimal behaviour in the natural habitat, I avoid the semantic subtleties of defining cognitive bias ${ }^{\dagger}$. Such behaviours violate 'ecological rationality' ${ }^{13}$ and, depending on interpretation, 'B-rationality'14. Rather than frequentist biases within a population (e.g., due to disorders ${ }^{6},{ }^{15},{ }^{16 *}$ ) or individual differences ${ }^{17}$, I focus on the more difficult topic of cognitive biases at the species level. These may sometimes be understood at a holistic level by taking account of not only the behaviours themselves, but the mechanisms that would be necessary for alternative (or 'better') behaviours to be supported.

I first discuss apparent 'explanations' that are flawed.

\section{Attempted explanations that do not address biased behaviours}

Various papers have attempted to explain sub-optimal cognitive biases from an evolutionary perspective. However, several aspects have caused confusion, resulting in some authors attempting to explain sub-optimal behaviours (such as hazardous decisions, assumed to be caused by a cognitive bias of overconfidence) using models where the behaviour is optimal. A brief history helps to clarify the issues.

An influential paper by Haselton et al. ${ }^{18,19^{* *}}$ identifies three classes of explanation for cognitive biases, which they term Heuristic, Error Management and Artifact biases. They describe heuristic biases as being due to information processing constraints (possibly due to phylogeny), resulting in mechanisms being used which fail to produce rational behaviour in systematic ways. Error management biases are produced by natural selection taking account not only of the probabilities of errors when taking particular actions, but the expected payoffs associated with those actions. Artifact biases are due to individuals being tested in non-natural settings, leading to non-rational processing of a problem.

The second of these, Error Management Biases, has received substantial attention. The mathematical basis of Error Management is signal detection theory ${ }^{20}$, which shows how signal distributions can be combined with payoffs to set optimal thresholds for behaviour. Unfortunately, in signal detection theory the optimal threshold setting referred to as the 'bias'. This label refers to the setting in terms of the probabilities, rather than payoffs (or utilities).

Actions often differ in expected costs and benefits, so optimal decisions need not minimise probability of error. This is easily seen by example. Suppose that you have the opportunity of gaining some money by calling the outcome of the flip a coin. For correctly calling heads, you win $f 2$; correctly calling tails, you win $f 1$; you get nothing for calling incorrectly. Using a fair coin, the expected payoff is maximised by calling heads. Even if the coin were slightly bent, so the probability of heads was only $40 \%$, the optimal behaviour is to call heads $\left(0.4^{*} £ 2>0.6^{*} f 1\right)$ even though the probability of calling correctly is maximised by calling tails. Looking only at the probabilities, rather than the expected payoffs, calling heads would appear biased.

\footnotetext{
${ }^{\dagger}$ My choice of definition would be a simplified version of that of Mineka \& Sutton (1992): 'a selective or nonveridical processing of information'.
} 
This kind of signal detection 'bias' produces optimal behaviour, so it is not correct to infer a suboptimal cognitive bias. This semantic confusion is exacerbated by it being easy to think in terms of local goals or probabilities, rather than overall utilities. For instance, Haselton et al. ${ }^{18}$ identify overperception of sexual interest (typically by males) as a cognitive bias, which can be explained simply by signal detection theory, as they recognise. (Similar examples abound in the animal kingdom ${ }^{21,22}$, including the possibility that some traits evolve to exploit existing cognitive biases in others ${ }^{23,24}$.) Although an interesting topic, and one that falls under a wide definition of cognitive bias $^{2 * *}$, this is not an explanation of sub-optimal behaviours (as identified by the authors ${ }^{25}$ and others $\left.{ }^{26}\right)$.

In combination with the semantic issue of bias, some authors have assumed a sub-optimal mental mechanism (i.e., a sub-optimal component of the decision-making system) and then inferred a cognitive bias by showing that the individual would do better if the mechanism treated probabilities in a biased manner. Without any basis for assuming such a mechanism in the first place, this is a nonsensical approach to trying to understand cognitive biases that produce sub-optimal behaviour; Marshall et al. ${ }^{27,28}$ correct some of this literature.

Others, such as Gigerenzer's ABC group, have suggested that we should not attempt to understand behaviours as optimal, arguing that optimal rules tend to be insufficiently robust. This argument is flawed as it has simply moved the goal-posts (if a rule were insufficiently robust, then it would not be optimal). I concur with Chater et al. ${ }^{29}$, who provide a strong rebuttal of several of the arguments against optimality modelling.

\section{How can we understand adapted biases that produce sub-optimal behaviours?}

A common explanation for sub-optimal behaviours (in the natural habitat) is phylogeny. If each tiny evolutionary modification must provide an advantage, then following a substantial environmental change (e.g., an ice-age), evolution can get stuck at points of local fitness maxima. It is also possible for this to occur in unchanging fitness landscapes and, at least in some cases, learning rules are unlikely to evolve without the environment altering over time ${ }^{30}$. There is a great difficulty with modelling this class of explanation however, as models are inherently abstract, whereas identifying the possible (mutational) paths through phylogenetic space typically requires a very detailed knowledge of the mechanisms. Thus, although the explanation has been used retrospectively for known physical 'design flaws' (such as the path of the laryngeal nerve), and likely also applies to some of the mental mechanisms that control behaviours, the phylogenetic approach does not provide a normative explanation of cognitive biases.

A related class of explanation, 'basins of attraction', also relates to fitness landscapes. By comparing classes of rule, it is sometimes possible to identify whether one class tends to operate well over a larger range of parameters. If so, then that rule is arguably more likely to be 'discovered' during evolution. This has been shown in theory for an example mental mechanism: the Rescorla-Wagner learning rule. The Rescorla-Wagner rule for the acquisition of associations is based on a trial-by-trial modification of associative strength as a function of the discrepancy between expected and realized outcome of each action. This model dominates the field of associative learning, but does not perform optimally (except under highly specific conditions). Trimmer et al. ${ }^{31}$ compare the RescorlaWagner rule with a class of rule that can achieve the optimal (Bayesian) outcome. Although the rule structure that supports the Bayesian optimum can, with finely tuned parameter values, outperform the best Rescorla-Wagner rule, the Rescorla-Wagner rule is far more likely to be discovered due to its larger basin of attraction (and subsequent fine tuning of parameters then makes it ever less likely that Bayesian rules emerge). 
An alternative, which I focus on for the rest of this paper, is that sub-optimal behaviour results from adaptive mental mechanisms. This concept requires us to separate the observer's perspective of optimal behaviour (in terms of a black-box approach of inputs, outputs and resultant payoffs) from a holistic view where internal costs (i.e., costs associated with the mental machinery) also govern the mental machinery that is available (i.e., has evolved) and the extent to which it is used.

\section{Holistically optimal vs behaviourally optimal}

It is simple to define the action of an individual as optimal if that action maximises expected return (given the data that has been received and prior expectations). I refer to such choices and actions as 'behaviourally optimal'.

Although the expected value of information should always be non-negative in a fully evolved system $^{32}$ (because the information would simply be ignored if processing it on average led to a reduced fitness), there can be substantial costs associated with processing information to make decisions. In humans for instance, brains take up only about $2 \%$ of body weight, but consume $20 \%$ of our oxygen at rest ${ }^{33}$ and even greater proportions of our glucose ${ }^{34}$. Taking account of such costs when considering possible actions, we see that there are two ways in which the actions of a 'holistically optimal' individual may differ from a behaviourally optimal individual.

First, an agent may rationally curtail their decision-making (i.e., restrict the use of some mental mechanisms) if processing is overly costly, or even choose situations that will be less mentally demanding ${ }^{35}$. Second, more fundamentally, benefits and costs will govern whether a mental mechanism even emerges as an adaptation than can be used. This can be seen easily by extending Paley's and Dawkins' topic of the evolution of a timekeeping mechanism ${ }^{36}$.

Suppose that an individual would benefit by measuring the passing of time (e.g., for rates of food reward, or photoperiod whilst preparing to migrate) and had a choice of three timepieces to carry in its head: a basic pendulum clock, a modern mechanical system, or an atomic clock. Knowing that an atomic clock costs a huge amount to make, is very heavy to carry around and requires a considerable amount of power on a continuous basis, the atomic clock should not be chosen. Although an individual choosing such a clock might make 'better' decisions when judged by an observer that was unaware of the internal costs, natural selection would act against such an individual. The decision between the simple but less accurate pendulum system, or the complicated (i.e., more costly and potentially risky) but more accurate watch would depend on many factors. The obvious point is that, even without phylogenetic constraints, the optimal choice will not result in individuals being able to measure time accurately so, from a behavioural perspective, they will often not perform optimally. Inaccuracies in timekeeping may result in non-symmetric costs (e.g., better to act early than late); compensating for this asymmetry can result in an apparent cognitive bias, despite the system operating optimally at the holistic level.

\section{Use of this approach}

The proportion of cognitive biases that can be accounted for using this approach is unclear at present. There will certainly be cases where phylogeny results in cognitive biases that correspond to local fitness maxima, and cases where relatively recent environmental changes are still being adapted to. However, this approach may provide significant insights into the evolution of mental constraints and thus sub-optimal behaviour. 
This approach is already shedding considerable light on the limitations of sensory systems. For instance, by comparing the properties of neurons in different types of fly, Niven et al. ${ }^{37}$ show how the costs and benefits of transporting a unit of information from the eye govern the visual properties that evolve. The authors also identify the fundamentally different types of costs involved (fixed irrespective of the amount of use - and variable). Such studies can help to inform future work by providing estimates for internal costs that are otherwise unknown. Although estimates of costs in larger networks are gradually becoming available (e.g., $38,39,40$ ) and insightful models of larger scale networks are emerging (e.g., ${ }^{41}$ ), a key remaining difficulty is knowing how costs scale relative to benefits in larger networks. By recognising this trade-off, researchers are making progress with certain types of disorders (e.g., ${ }^{42}$ ); the same approach can also be used regarding cognitive biases. If a species does not have sufficient memory capacity to deal with all situations optimally, even in natural settings, it may be that the internal costs associated with such capacity would be restrictive; under such circumstances, we should not expect behaviour to appear optimal to an observer, but to be optimal at the holistic level.

The incorporation of mental costs in decision-making processes may also shed light on some wellestablished problems, such as Allais' paradox ${ }^{43}$, which indicates that expected utility theory does not hold, thus suggesting a cognitive bias. Results such as these have led to highly influential theories, such as prospect theory ${ }^{44}$ that are non-normative (though see ${ }^{45}$ ). I show how mental costs may account for this finding in Appendix $A$, including a method of testing the concept.

\section{Difficulties and potential for confusion}

There are various ways in which confusion could arise when incorporating internal costs into models. Due to the costs of mechanisms, a single mechanism may be used for multiple tasks; this may lead to cognitive biases in some tasks. However, to model this, the multiple functional uses of a mechanism must be described, along with alternative possibilities for producing behavioural responses; this is not easy. Similarly, a task may be dealt with by one of several applicable mechanisms, depending on the current parameters of that task (e.g., ${ }^{46,47}$ ); this again makes it more difficult to build predictive models, as there is difficulty in abstracting any single mental mechanism in relation to a task. Nevertheless, it also provides a route into explaining cognitive biases such as framing effects ${ }^{48}$.

Other difficulties include choosing the appropriate internal costs of mechanisms when there are many costs to choose from: the fixed and variable energy costs of running a mechanism, resource costs of building and maintenance, processing time, storage costs of memory, and how the risk of malfunction increases with complexity.

If a behaviour is very close to optimal, then it might be assumed either that the cost of a single mistake is substantial, or the decision is required often (so smaller payoff differences accumulate). However, knowing that mental costs mean that mechanisms are often used for more than one purpose, a third possibility emerges: if a particular decision (to a particular type of problem, A) requires inputs from more than one mental mechanism, then if those mental mechanisms have strong selection pressures on them for other purposes (e.g., problems $B$ and $C$ ), then the nearoptimal behaviour (under problem A) may arise as a by-product of the other selection pressures. Thus, near-optimal behaviour may, counterintuitively, arise as a by-product in some circumstances.

\section{Discussion}

Although much useful work has been done by building models of optimal behaviour, I am arguing that the more fundamental trade-off relates to the decision-making mechanisms that evolve: the trade-off between the many internal (often hidden) costs of the machinery and the benefits that 
they produce. In cases where the internal costs are small, we can still expect the system to produce behaviour that appears very close to optimal (i.e., without cognitive bias).

The general thrust of this paper is, in some ways, not new. McNamara \& Houston ${ }^{49}$ highlighted the need to identify simple mechanisms that work well in complex environments. I suggest that, to a large extent, the need for mechanisms to be simple reflects the inherent costs associated with complex mechanisms. Similarly, Fawcett et al..$^{5 *}$ have identified that many authors assume the 'behavioural gambit' despite learning and decision-making not always producing optimal behaviour; they conclude that "we would do better by considering the underlying mechanisms, rather than the behavioral outcomes they produce, as the target of selection." I concur with Fawcett at al., and suggest a route ahead of taking internal costs into account when considering issues such as cognitive biases. In the longer-term, these hidden costs associated with computation may also provide a route to understanding consciousness and culture ${ }^{51}$.

Recent simulation models have assumed particular mental architectures for decision making. For instance, based on empirical findings, Eliassen et al..$^{52}$ have assumed that an individual has a 'global organismic state' of being hungry or fearful. This provides useful insights into how such an organism reacts under different circumstances, but cannot tell us why such a global organismic state exists in this form.

By making the simplifying assumption that any mental mechanism could evolve (the phenotypic gambit ${ }^{53}$ ) but imposing costs on mental processes and allowing cognitive constraints to emerge, we may obtain a normative explanation for some cognitive biases. This would be preferable to assuming that such biases occur through phylogeny, as it allows such behaviours to be understood from all angles ${ }^{54}$. However, this functional explanation is no longer at the behavioural level described by Tinbergen; this approach shifts the explanation to the costs and benefits associated with mental machinery rather than individual behaviours produced by that machinery.

\section{Acknowledgements}

This work was supported by the NSF (IOS 1456724 grant to A. Sih). I thank Alasdair I. Houston and John M. McNamara for useful discussions around this topic in the past, and Alex Kacelnik and an anonymous referee for useful feedback on this ms.

\section{References}

1. Tversky, A. \& Kahneman, D. (1974) Judgment under Uncertainty: Heuristics and Biases. Science 185, Issue 4157, 1124-1131.

2. Harding, E.J., Paul, E.S., Mendl, M. (2004) Cognitive bias and affective state. Nature 427, 312.

3. Scollo, A, Gottardo, F., Contiero, B., Edwards, S.A. (2014) Does stocking density modify affective state in pigs as assessed by cognitive bias, behavioural and physiological parameters? Applied Animal Behaviour Science 153, 26-35.

4. Bateson, M. \& Nettle, D. (2015) Development of a cognitive bias methodology for measuring low mood in chimpanzees. PeerJ 3:e998

5. Raghubir, P. and Ranjan, S. (1999) The Psychology of Financial Decision Making: A Case for Theory-Driven Experimental Enquiry.

6. *Clark, L., Studer, B., Bruss, J., Tranel, D., Bechara, A. (2014) Damage to insula abolishes cognitive distortions during simulated gambling. PNAS 111 (16) 6098-6103. 
This paper looks at the effect of brain damage on cognitive biases, such as the gambler's fallacy, in human subjects. The results suggest that people who suffer from problem gambling may be making too much use of one part of the brain, exaggerating a cognitive bias which then encourages them to see patterns where there are none and thus encouraging them to gamble.

7. Barrus, M.M., Hosking, J.G., Cocker, P.J., Winstanley, C.A. (2016) Inactivation of the orbitofrontal cortex reduces irrational choice on a rodent betting task. Neuroscience http://dx.doi.org/10.1016/j.neuroscience.2016.02.028

8. Zalocusky, K.A., Ramakrishnan, C., Lerner, T.N., Davidson, T.J., Knutson, B., Deisseroth, K. (2016) Nucleus accumbens D2R cells signal prior outcomes and control risky decision-making. Nature $531,642-646$.

9. *Soltani, A., Khorsand P., Guo, C., Farashahi, S., Liu, J. (2016) Neural substrates of cognitive biases during probabilistic inference. Nature Communications 7:11393.

This paper is aimed at the neural mechanisms underlying cognitive biases. The authors build biologically plausible models and contrast them with empirical data that are not normative (at least, without assuming internal costs). The work provides compelling evidence for cognitive biases arising through the interactions of sub-systems in the brain.

10. Gigerenzer, G. \& Fiedler, K. (2003) Minds in Environments: The potential of an ecological approach to cognition. Submitted for publication; available online.

11. Charnov, E.L. (1976) Optimal foraging: the marginal value theorem. Theoretical Population Biology 9, 129-136.

12. Laming, D.R.J. (1968) Information theory of choice-reaction times. Academic Press, Oxford.

13. Hutchinson, J.M.C. \& Gigerenzer, G. (2005) Simple heuristics and rules of thumb: where psychologists and behavioural biologists might meet. Behav. Proc. 69, 97-124.

14. Kacelnik A. (2006) Meanings of rationality. In: Rational Animals? (Eds S. Hurley and M. Nudds). Oxford University Press: Oxford.

15. Belzung, C., Willner, P., Philippot, P. (2015) Depression: from psychopathology to pathophysiology. Current Opinion in Neurobiology 30, 24-30.

16. *O'Callaghan, C., Kveraga, K., Shine, J.M., Adams Jr., R.B., Bar, M. (2016) Predictions penetrate perception: Converging insights from brain, behaviour and disorder. Consciousness and Cognition; available online: doi:10.1016/j.concog.2016.05.003.

Visual perception is a two-way street of top-down predictions governing attention whilst data (bottom up) continues to arrive. The authors review behavioural and mechanistic evidence, showing that many factors, including cognitive biases, affect perception. The factors, whilst taken into account in a balanced way in healthy brains, may not be balanced in unhealthy individuals (e.g., those that suffer from hallucinations).

17. Teovanović P., Knežević, G., Stankov, L. (2015). Individual differences in cognitive biases: Evidence against one-factor theory of rationality. Intelligence, 50, 75-86.

18. Haselton, M.G., Nettle, D., Andrews, P.W. (2005) The evolution of cognitive bias. In D.M. Buss (Ed.), The handbook of evolutionary psychology. New York: Wiley.

19. ${ }^{* *}$ Haselton, M.G., Nettle, D., Murray, D.R. (2015) The evolution of cognitive bias. In D.M. Buss (Ed.), The handbook of evolutionary psychology. $2^{\text {nd }}$ edition. New York: Wiley.

This paper is an update from the highly influential 2005 paper, incorporating a definition of cognitive bias. It is influential in terms of both in terms of semantics and defining evolutionary approaches to explaining such biases. The authors identify three reasons for finding apparent design flaws: 'heuristics', 'error management biases' and 'artifacts'. I concur with their general approach (e.g., heuristics are likely to be selected for due to reduced internal costs, artefact biases can arise through phylogenetic constraints) but would not label optimal behaviour (explained by the management of errors) a real/true cognitive bias.

20. Green, D.M. and Swets, J.A. (1966) Signal Detection Theory and Psychophysics. Wiley and Sons, NY. 
21. Takeshita, F. \& Sato, N (2016) Adaptive Sex-Specific Cognitive Bias in Predation Behaviours of Japanese Pygmy Squid. Ethology 122(3), 236-244.

22. Blanchard, T.C., Wilke, A., Hayden, B.Y. (2014) Hot-hand bias in rhesus monkeys. Journal of Experimental Psychology: Animal Learning and Cognition 40(3), 280-286.

23. Castellano, S. \& Cermelli, P. (2015) Preys' exploitation of predators' fear: when the caterpillar plays the Gruffalo. Proc. R. Soc. B 282: 20151786.

24. Peterson, R.L. (2016) The Only Thing to Fear. Chapter in Trading on Sentiment, published online by Wiley. DOI: 10.1002/9781119219149.ch9

25. Haselton, M.G. \& Nettle, D. (2006) The paranoid optimist: an integrative evolutionary model of cognitive biases. Pers. Soc. Psychol. Rev. 10, 47-66

26. McKay, R. \& Efferson, C. (2010) The subtleties of error management. Evolution and Human Behavior 31(5), 309-319.

27. Marshall, J.A.R., Trimmer, P.C., Houston, A.I., McNamara, J.M. (2013) On evolutionary explanations of cognitive biases. TREE 28(8), 469-473.

28. Marshall, J.A.R., Trimmer, P.C., Houston, A.I. (2013b) Unbiased individuals use valuable information when making decisions: a reply to Johnson and Fowler. TREE 28(8), 444-445.

29. Chater, N., Oaksford, M., Nakisa, R., Redington, M. (2003) Fast, frugal, and rational: How rational norms explain behaviour. Organizational Behaviour and Human Decision Processes 90, 63-86.

30. Trimmer, P.C. \& Houston, A.I. (2014) An evolutionary perspective on information processing. Topics in Cognitive Science, 1-19.

31. Trimmer, PC, McNamara, JM, Houston, AI, Marshall, JAR (2012) Does natural selection favour the Rescorla-Wagner rule? J Theor Biol 302, 39-52.

32. McNamara, J.M. \& Dall, S.R.X. (2010) Information is a fitness enhancing resource. Oikos 119, 231-236.

33. Clarke, D.D. \& Sokoloff, L. (1999) Basic Neurochemistry: Molecular, Cellular and Medical Aspects. 6th edition. Lippincott-Raven, Philadelphia.

34. Kuzawa, C.W., Chugani, H.T., Grossman, L.I., Lipovich, L., Muzik, O., Hof, P.R., Wildman, D.E., Sherwood, C.C., Leonard, W.R., Lange, N. (2014) Metabolic costs and evolutionary implications of human brain development. PNAS 111(36), 13010-13015.

35. Hosking, J.G., Cocker, P.J., Winstanley, C.A. (2015) Prefrontal cortical inactivations decrease willingness to expend cognitive effort on a rodent cost/benefit decision-making task. Cereb Cortex. 1-10.

36. Dawkins, R. (1986) The Blind Watchmaker. Norton \& Company, Inc. NY.

37. Niven, J.E., Anderson, J.C., Laughlin, S.B. (2007) Fly photoreceptors demonstrate energyinformation trade-offs in neural coding. PLoS Biol 5(4): e116.

38. Attwell, D. \& Laughlin, S.B. (2001) An energy budget for signaling in the grey matter of the brain. J Cereb Blood Flow Metab 21(10), 1133-1145.

39. Laughlin, S.B. (2011) Energy, information, and the work of the brain. In: Work Meets Life. MIT Press, Cambridge, Mass. 39-67.

40. Snell-Rood, E.C., Papaj, D.R., Gronenberg, W. (2009) Brain Size: A Global or Induced Cost of Learning? Brain, Behavior and Evolution 73, 111-128.

41. Komer, B. \& Eliasmith, C. (2016) A unified theoretical approach for biological cognition and learning. Current Opinion in Behavioural Sciences, available online 19 March 2016.

42. Bullmore, E. \& Sporns, O. (2012) The economy of brain network organization. Nature Reviews Neuroscience 13, 336-349.

43. Allais, M. (1953) Le comportement de l'homme rationnel devant le risque: critique des postulats et axiomes de l'école Américaine. Econometrica 21(4), 503-546.

44. Kahneman, D. \& Tversky, A. (1979) Prospect Theory: an analysis of decision under risk. Econometrica 47(2), 263. 
45. Mallpress, D.E.W., Fawcett, T.W., Houston, A.I., McNamara, J.M. (2015) Risk attitudes in a changing environment: an evolutionary model of the fourfold pattern of risk preferences. Psychological Review 122(2), 364-375.

46. Carpenter, R.H.S. \& Williams, M.L.L. (1995) Neural computation of log likelihood in control of saccadic eye movements. Nature, 377(6544), 59-62.

47. Mobbs, D., Petrovic, P., Marchant, J.L., Hassabis, D., Weiskopf, N., Seymour, B., Dolan, R.J. \& Frith, C.D. (2007) When fear is near: threat imminence elicits prefrontal-periaqueductal gray shifts in humans. Science 317, 1079-1083.

48. Kahneman, D. \& Tversky, A. (1981) The framing of decisions and the psychology of choice. Science 211(4481), 453-458.

49. McNamara, J.M. \& Houston, A.I. (2009) Integrating function and mechanism. Trends in Ecology and Evolution 24(12), 670-675.

50. *Fawcett, T.W., Hamblin, S., Giraldeau, L.-A. (2013) Exposing the behavioral gambit: the evolution of learning and decision rules. Behav Ecol 24 (1): 2-11.

This paper makes a useful distinction between the phenotypic gambit (that any morphology can evolve) and the 'behavioural gambit', that any behaviour can evolve. Although the behavioural gambit has often been assumed, the authors provide several examples where behaviours appear sub-optimal. They highlight that we should focus on the evolution of mental mechanisms, rather than behaviours directly, and provide a different emphasis on the way forward to that proposed in this paper.

51. Thompson, B., Kirby, S., Smith, K. (2016) Culture shapes the evolution of cognition. PNAS 45304535.

52. Eliassen, S., Andersen, B.S., Jørgensen, C., Giske, J. (2016) From sensing to emergent adaptations: modelling the proximate architecture for decision-making. Ecological Modelling 326, 90-100.

53. Grafen, A. (1984) Natural selection, kin selection and group selection. In: (Krebs JR, Davies NB, eds) Behavioural ecology: an evolutionary approach, 2nd edition. Blackwell Scientific Press, Oxford, UK. 62-84.

54. Tinbergen, N. (1963) On aims and methods in ethology. Zeitschrift fur Tierpsychologie 20, 410433.

55. Wikipedia (2016) https://en.wikipedia.org/wiki/Cognitive_bias Downloaded 21 Jan 2016.

56. Mendl, M., Burman, O.H.P., Parker, R.M.A., Paul, E.S. (2009) Cognitive bias as an indicator of animal emotion and welfare: Emerging evidence and underlying mechanisms. Applied Animal Behaviour 118, Iss 3-4, 161-181.

57. Mineka, S. \& Sutton, S.K. (1992) Cognitive biases and the emotional disorders. Psychological Science 3(1), 65-69.

58. Mineka, S. \& Tomarken, A.J. (1989) The role of cognitive biases in the origins and maintenance of fear and anxiety disorders. In T. Archer \& L.-G. Nilsson (Eds) Aversion, avoidance and anxiety: Perspectives on aversively motivated behaviour (pp. 195-221) Hillsdale, NJ: Erlbaum.

\begin{tabular}{|l|l|}
\hline \multicolumn{1}{|c|}{ Definition } & \multicolumn{1}{c|}{ Semantics/Issues } \\
\hline $\begin{array}{l}\text { Cases in which human cognition } \\
\text { reliably produces representations that } \\
\text { are systematically distorted compared } \\
\text { to some aspect of objective reality. }{ }^{19^{* *}}\end{array}$ & $\begin{array}{l}\text { Any behaviour that is not reward-maximising could } \\
\text { indicate a cognitive bias, even in non-natural lab } \\
\text { settings. Thus, removing the term 'human' from the } \\
\text { definition to study other animals, we find that an } \\
\text { animal responding optimally (relative to its natural } \\
\text { setting) may be regarded as cognitively biased. }\end{array}$ \\
\hline An inaccurate view of the world. ${ }^{27}$ & $\begin{array}{l}\text { Any behaviour that is not reward-maximising could } \\
\text { indicate a cognitive bias, even in non-natural lab } \\
\text { settings. }\end{array}$ \\
\hline
\end{tabular}




\begin{tabular}{|l|l|}
\hline $\begin{array}{l}\text { A systematic pattern of deviation from } \\
\text { norm or rationality in judgment, } \\
\text { whereby inferences about other people } \\
\text { and situations may be drawn in an } \\
\text { illogical fashion. }\end{array}$ & $\begin{array}{l}\text { As } \\
\text { 'rational' means, adaptive behaviour may not be } \\
\text { regarded as cognitively biased. }\end{array}$ \\
\hline $\begin{array}{l}\text { Effects of emotional state or trait on } \\
\text { cognitive processes. }\end{array}$ & $\begin{array}{l}\text { Any behaviour can be inferred to show a cognitive bias } \\
\text { if emotions are deemed to be a part of the decision- } \\
\text { making process. }\end{array}$ \\
\hline $\begin{array}{l}\text { Any selective or nonveridical } \\
\text { processing of emotion-relevant } \\
\text { information (57, based on a longer } \\
\text { version, }{ }^{58} \text {, specific to fear and anxiety). }\end{array}$ & $\begin{array}{l}\text { It is unclear why the authors included 'emotion- } \\
\text { relevant' in their definition, except that they were } \\
\text { dealing with emotions in their paper. }\end{array}$ \\
\hline $\begin{array}{l}\text { Interesting cognitive biases obtain } \\
\text { when beliefs depart systematically } \\
\text { from those of an agent with Bayesian } \\
\text { beliefs. }\end{array}$ & $\begin{array}{l}\text { The authors point out that just about any decision- } \\
\text { maker does not assign equal probability to every } \\
\text { possibility - and that to do so would be a mistake. }\end{array}$ \\
\hline
\end{tabular}

Table 1: The term 'cognitive bias' has somewhat different meanings. 